\title{
A systematic review of pathophysiology and management of familial hyperaldosteronism type 1 in pregnancy
}

\author{
Viola Sanga $\mathbb{(}^{1,2} \cdot$ Teresa Maria Seccia $\mathbb{i}^{1} \cdot$ Gian Paolo Rossi $\mathbb{C}^{1}$
}

Received: 18 April 2021 / Accepted: 10 May 2021 / Published online: 27 May 2021

(c) The Author(s) 2021

\begin{abstract}
Purpose Familial hyperaldosteronism type $1(\mathrm{FH}-1)$ is a rare autosomal dominant form of primary aldosteronism, which features a marked phenotypic heterogeneity, ranging from mild to severe forms of arterial hypertension that can be complicated by stroke and cardiovascular events at a young age. As affected patients usually reach the fertile age, transmission of the disease to offspring is common. Notwithstanding this, reports of FH-1 in pregnancy are limited and there is a lack of treatment guidelines.

Methods and results We searched the PubMed and EuropePMC databases with a PICO strategy to retrieve available information on management of FH-1 patients during pregnancy. We could identify seven relevant articles, which are herein reviewed. Conclusion Based on available information on pathophysiology and treatment of FH-1 in pregnancy, recommendations for the rational management of FH-1 in pregnancy are provided.
\end{abstract}

Keywords Hypertension • Pregnancy • Primary Hyperaldosteronism • Familial Hyperaldosteronism type $1 \cdot$ Glucocorticoidremediable aldosteronism

\section{Introduction}

In 1969, a familial form of primary aldosteronism (PA) relieved by dexamethasone treatment was reported and defined glucocorticoid-remediable-aldosteronism (GRA) (OMIM: 103900) [1, 2]. As additional forms of familial hyperaldosteronism (FH) were thereafter identified [3-6], GRA was thereafter designated as FH type 1 (FH-1) [7].

FH-1 is transmitted as an autosomal dominant disorder and is characterized by a marked phenotypic heterogeneity. Reported clinical phenotypes ranged from mild arterial hypertension and aldosteronism to severe forms complicated

Supplementary information The online version contains supplementary material available at https://doi.org/10.1007/s12020021-02763-5.

Gian Paolo Rossi

gianpaolo.rossi@unipd.it

1 Hypertension and Emergency Unit, Department of Medicine DIMED, University of Padua, Padova, Italy

2 PhD Arterial Hypertension and Vascular Biology, Department of Medicine - DIMED, University of Padua, Padova, Italy by cardiovascular events, mainly ischemic or hemorrhagic strokes, occurring at a young age [8, 9].

In 1992, Lifton's laboratory identified a chimeric gene [10] resulting from homologous recombination between the genes encoding 11- $\beta$ hydroxylase (CYP11B1) and aldosterone synthase (CYP11B2). These genes share 95\% homology and map close to each other on chromosome $8 q 24$. In the chimeric gene, acquisition of the adrenocorticotropic hormone (ACTH) responsive elements of CYP11B1, along with the CYP11B2 sequences encoding aldosterone synthase, explained both the ectopic expression of aldosterone synthase in the adrenocortical zona fasciculata, which normally secretes cortisol, and the correction of FH-1, i.e., resolution of the hyperaldosteronism and normalization of the high blood pressure (BP) values, with suppression of the ACTH-drive by glucocorticoid treatment.

This discovery lead to develop genetic testing based on long polymerase chain reaction (PCR) [11], which permits a conclusive diagnosis of $\mathrm{FH}-1$, and therefore, institution of glucocorticoid treatment. $\mathrm{FH}-1$ is rare in that it affects $<1 \%$ of PA patients [12, 13]. However, since PA is common in hypertensive patients [14, 15], but often overlooked, the prevalence rate of $\mathrm{FH}-1$, particularly of its milder forms, likely is markedly underestimated, as most FH-1 patients present with mild clinical PA phenotypes. Therefore, FH-1 
patients usually reach the fertile age, which allows transmission of the disease to offspring. However, pedigrees entailing fatal strokes and aortic rupture occurring at a young age have also been reported [9]. In spite of this, information on pathophysiology and management of FH-1 in pregnancy is scant [16-19] and treatment guidelines lack. Hence, we have searched in the literature for available information on pathophysiology and course of FH-1 in pregnancy, with the final goal of providing some recommendations for the management of this condition.

\section{Methods}

We searched the PubMed and EuropePMC databases with a PICO strategy (Table S1) to identify available information/ papers on the best management of FH-1 patients during pregnancy.

The PRISMA strategy was then applied using the following boolean operators: ['Familial hyperaldosteronism type 1' OR 'Glucocorticoid remediable aldosteronism'] AND ['pregnant' OR 'pregnancy'] (Fig. S1).

\section{Results}

In total, 400 papers containing the searched terms (PRISMA graph Fig. S1) were found, but only seven comprising anecdotal cases were judged to be relevant for the management of FH-1 patients during pregnancy (Table 1 and Table S2), indicating that systematic research efforts are needed on this topic.

In 1999 a retrospective study of the maternal and fetal outcomes of 35 pregnancies in 16 women with proven FH-1, albeit of undetermined severity, concluded that in FH-1 women there was no evidence for an excess incidence of preeclampsia, which occurred at a rate $(6 \%)$ well within the range (between 2.5 and 10\%) seen in the general obstetric population [18]. No cases of overt eclampsia, or the syndrome of hemolysis, elevated liver function tests, and low platelets (HELLP) were seen. In hypertensive women an exacerbation of hypertension during gestation occurred in $39 \%$ of the pregnancies and resolution of hypertension was reported in $17 \%$ of the pregnancies. It remained uncertain when resolution occurred, because the study did not report the time course of hypertension. Of note, $23 \%$ of the pregnancies $(n=8)$ required one or more antihypertensive medications; all these women were reported to have hypertension before pregnancy and $17 \%$ were on antihypertensive medications from the onset of pregnancy. A cesarean section was performed in $43 \%$ of the pregnancies. A trend toward a lower weight at birth was seen in the infants of the FH-1 mothers who experienced pregnancy-aggravated hypertension.
In 2002, 29 pregnancies in eight FH-1 women were reported in a five-generation $\mathrm{FH}-1$ benign pedigree with a mild phenotype entailing a high number of normotensive or mildly hypertensive members and a much lower frequency of stroke than in other FH-1 pedigrees [19]. No pregnancies were complicated by preeclampsia and no cesarean sections were performed, indicating an uneventful course of pregnancy in this pedigree.

In the following decades only two other cases of FH-1 in pregnancy were reported [16, 17]. Hamilton et al. [17] described a woman from a pedigree with young onset hypertension and stroke, who presented at age 28 years with high BP $(160 / 90 \mathrm{mmHg})$. The woman and her father tested positive for FH-1. She was treated with the nondihydropiridine calcium channel blocker verapamil, after which she became pregnant and normotensive and verapamil could be withdrawn. Notwithstanding this, her BP values remained normal without medications throughout pregnancy. Her plasma renin activity (PRA) progressively rose to the upper normal range of $2.7 \mathrm{ng} / \mathrm{ml} / \mathrm{h}$ at week 18 th and to elevated values $(7.0 \mathrm{ng} / \mathrm{ml} / \mathrm{h})$ at week 24 th of gestation, while plasma aldosterone concentration (PAC) values fell within the normal range $(260 \mathrm{pmol} / \mathrm{L}$ equivalent to $9.4 \mathrm{ng} / \mathrm{dl}$ ) at week $18 \mathrm{th}$, and then became elevated (685 $\mathrm{pmol} / \mathrm{L}$ equivalent to $24.7 \mathrm{ng} / \mathrm{dl}$ ) at week 24 th. Information on delivery and/or newborn outcome were missing. At 1month postpartum, the BP values raised to $160 / 120 \mathrm{mmHg}$; PRA was suppressed $(0.29 \mathrm{ng} / \mathrm{ml} / \mathrm{h})$ and PAC remained overtly high $(605 \mathrm{pmol} / \mathrm{L}$ equivalent to $21.8 \mathrm{ng} / \mathrm{dl})$, resulting in an unambiguously elevated ARR of $90 \mathrm{ng} / \mathrm{mIU}$ (n.v. < $20.6 \mathrm{ng} / \mathrm{mIU})$. Prednisolone treatment was commenced at $2.5 \mathrm{mg}$ daily, but whether BP values normalized with such treatment was not reported.

In 2014, Campino et al. [16] described a 21 years-old FH-1 pregnant woman from a FH-1 family. At age 16, her ARR was high $(70 \mathrm{ng} / \mathrm{mIU})$; after 3 months of dexamethasone treatment $(0.25 \mathrm{mg}$ o.d.) both high BP and ARR values were normalized. At age 21 she became pregnant and discontinued the drug. Her BP was normal throughout gestation with no antihypertensive medications, with values ranging between 103/69 and 110/70 $\mathrm{mmHg}$. However, at week 28th her ARR was borderline elevated $(20 \mathrm{ng} / \mathrm{mIU})$; at week 36 th it increased to a value, which was consistent with PA ( $30 \mathrm{ng} / \mathrm{mIU})$. The ARR rose further to $240 \mathrm{ng} / \mathrm{mIU}$ in the postpartum period, a rise that was accompanied by only a modest increase of BP to an average of 136/94 mmHg. Further information on treatment, BP and ARR values was missing.

We recently reported on a 31 years-old Caucasian pregnant FH-1 woman from a severe FH-1 pedigree [20] featuring a very high incidence of stroke and premature aortic rupture. At the time of presentation, her ARR, as calculated with the ARR-App [21], was more than 10-fold 


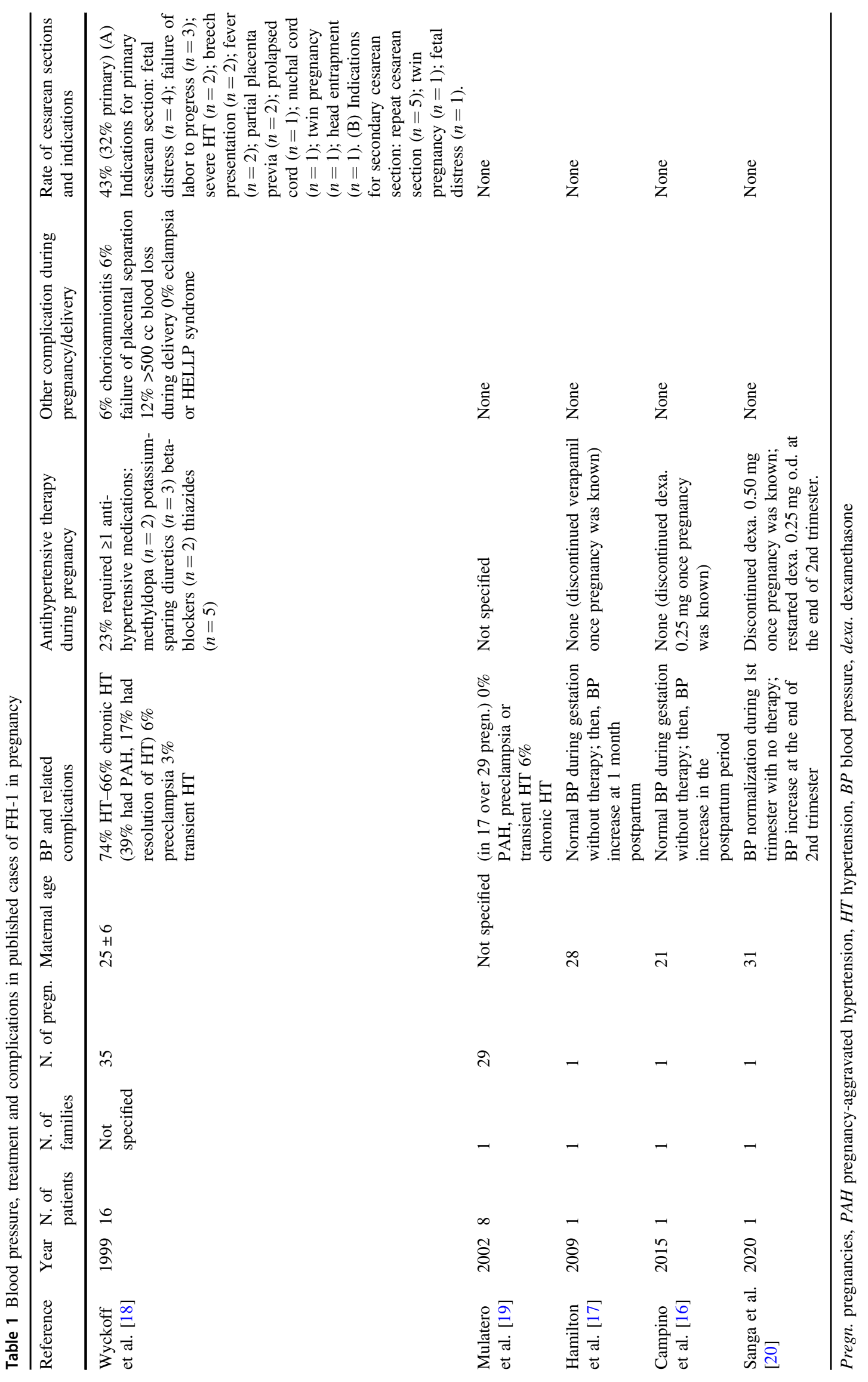


higher than the upper normal range ( $290 \mathrm{ng} / \mathrm{mIU}$; n.v. <20.6). After confirming the diagnosis of FH-1 by genetic testing, control of arterial hypertension and hyperaldosteronism was accomplished with low-dose dexamethasone $(0.50 \mathrm{mg}$ o.d. in the evening) treatment. Four years after presentation she became pregnant and was advised to stop dexamethasone treatment and to undertake close monitoring of BP and serum $\mathrm{K}^{+}$values. At the end of the 2 nd trimester her hypertension recurred and she was restarted on dexamethasone $(0.25 \mathrm{mg}$ o. d. in the evening), which rapidly normalized her high BP values. She delivered a healthy normal weight male baby at term with no complications whatsoever. The postnatal course was uneventful and the newborn growth was normal. After delivery, she restarted dexamethasone $(0.50 \mathrm{mg}$ o.d.) with persistent normalization of BP and PAC values. She refused to provide the baby's DNA for a free genetic test for FH-1 notwithstanding multiple invitations. Five years later she informed us that her 5-years old child had developed severe drug-resistant hypertension, but did not accept an invitation for a free consultation.

\section{Discussion}

Several pathophysiological considerations need to be made concerning FH-1 in pregnancy. During normal pregnancy, progesterone and aldosterone increase in parallel and this rise is held to be key for the normal development of the placenta [22]. Nonetheless, aldosterone secretion remains tightly regulated in pregnancy, which allows the mother to cope with changes of salt intake, and to develop volume expansion during pregnancy [23].

ACTH and cortisol levels also increase during pregnancy secondary to placental production of corticotropin releasing hormone (CRH) [24]. Given that that aldosterone production is ACTH-dependent in FH-1, it was, therefore, hypothesized that the ACTH increase could exacerbate hyperaldosteronism and hypertension during FH-1 pregnancy [23]. However, since progesterone acts as a mineralocorticoid receptor antagonist, its raised levels in pregnancy may block the effects of the increased aldosterone [25].

Furthermore, progesterone, but not estradiol, was reported to inhibit the chimeric isoforms of aldosterone synthase in HEK-293 cells transiently transfected with vectors containing the full chimeric CYP11B2 cDNAs [26], thus suggesting a peculiar beneficial action of this hormone in $\mathrm{FH}-1$ pregnancy. Collectively, the available results point to the increase of progesterone as a plausible mechanism of $\mathrm{BP}$ and PAC normalization during the first trimester of pregnancy and throughout pregnancy, as noted in the two aforementioned clinical cases [16, 17], where
Fig. 1 Diagnostic and therapeutic algorithm for screening and management of women with hypertension and possible FH-1. ARR aldosterone-renin ratio, HT hypertension PA primary aldosteronism
Fertile women with HT who consider pregnancy

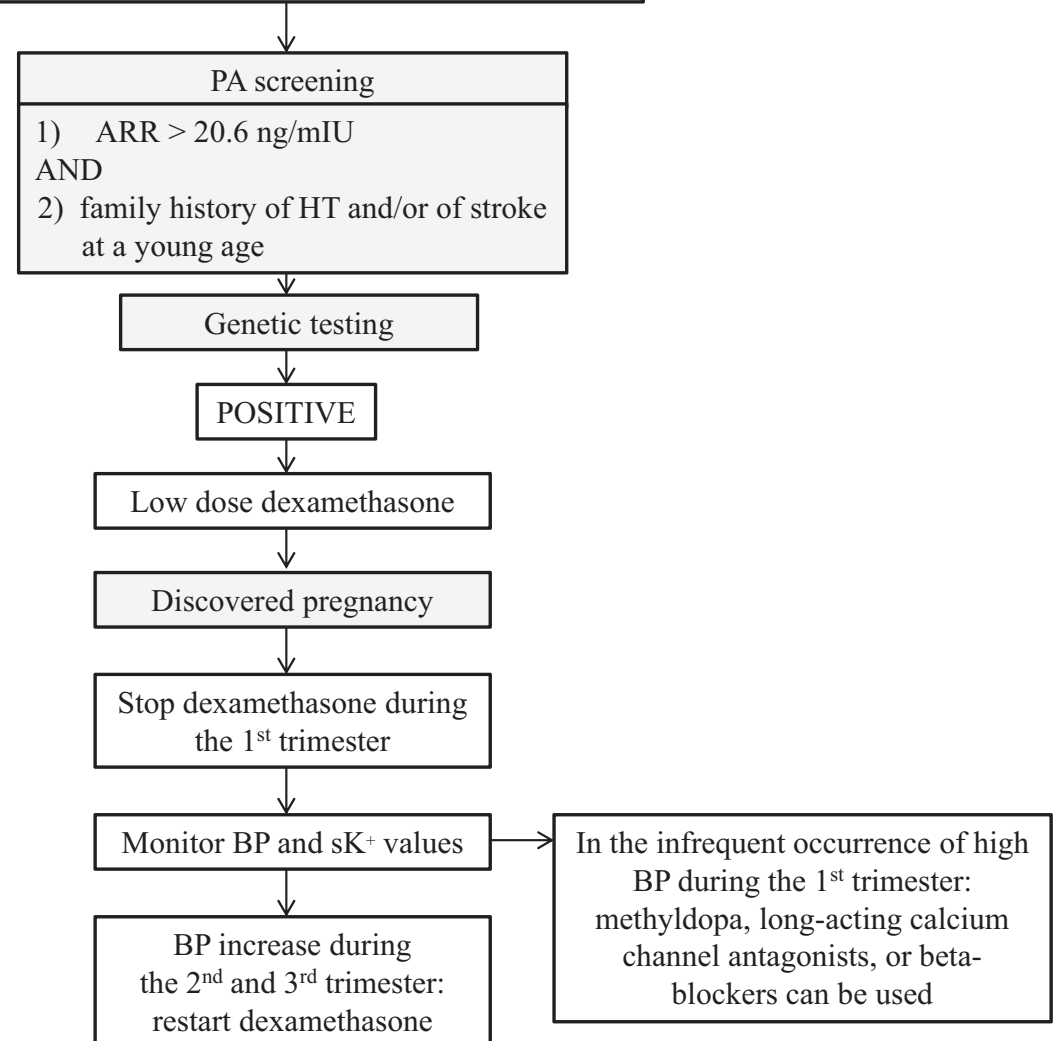


pharmacological therapy was not necessary during pregnancy, and also up to the third trimester in the most recent case [20].

As regards treatment of FH-1 women who become aware of being pregnant during the 1 st trimester, immediate withdrawal of dexamethasone with careful monitoring of BP values and serum $\mathrm{K}^{+}$levels is advised, because of its potential negative effects on fetal development. However, it is worth noting that dexamethasone is the most potent and long-acting glucocorticoid drug in suppressing ACTH [27]. It was also contented that prednisolone and hydrocortisone, being inactivated by placental 11- $\beta$-hydroxysteroid dehydrogenase type 2 (11- $\beta$-HSD-2) more effectively than dexamethasone, should be preferred during pregnancy. However, since treatment of FH-1 during pregnancy is for the mother and not for the fetus, it seems logical to select the most potent ACTH-suppressive treatment with dexamethasone and prefer the lowest dose that warrants normotension and control of hypokalemia in case of BP values increase and serum $\mathrm{K}^{+}$levels fall during the 3 rd trimester. Exploitation of such strategy in the last case observed [20], where the effectiveness of low-dose dexamethasone was well-documented before pregnancy, warranted not only an uneventful course of gestation, but also delivery of a healthy baby, albeit likely also affected by FH-1.

In conclusion, based on the available information in the literature, the following recommendations for the management of FH-1 women in pregnancy can be put forward. All fertile young women with arterial hypertension who consider pregnancy, should be screened beforehand for PA as indicated (Fig. 1) [28]. A raised ARR should be followed by a search for the chimeric gene in all women who have a strong family history of hypertension and/or stroke at young age. In FH-1 women who become aware of being pregnant, immediate withdrawal of dexamethasone followed by careful monitoring of $\mathrm{BP}$ values and serum $\mathrm{K}^{+}$levels is advised during the 1 st trimester. Should blood pressure become uncontrolled during this trimester, the drugs that are usually prescribed in hypertensive women during pregnancy including methyldopa, long-acting calcium channel antagonists, or beta-blockers [29], can be used. If BP rises during the 2 nd and/or the 3rd trimester, low to very low dose dexamethasone is the treatment of choice with the aim of normalizing BP but also PAC, renin and serum $\mathrm{K}^{+}$ levels.

Acknowledgements Dr. Sanga collected data from literature and drafted the manuscript that was extensively revised and commented on by Prof. Rossi and Prof. Seccia.

Funding Grant support to Prof. Rossi: FORICA (The FOundation for advanced Research In Hypertension and CArdiovascular diseases), the University of Padua and The International PhD Program in Arterial Hypertension and Vascular Biology.
Funding Open access funding provided by Università degli Studi di Padova within the CRUI-CARE Agreement.

\section{Compliance with ethical standards}

Conflict of interest All authors have no competing interests.

Publisher's note Springer Nature remains neutral with regard to jurisdictional claims in published maps and institutional affiliations.

Open Access This article is licensed under a Creative Commons Attribution 4.0 International License, which permits use, sharing, adaptation, distribution and reproduction in any medium or format, as long as you give appropriate credit to the original author(s) and the source, provide a link to the Creative Commons license, and indicate if changes were made. The images or other third party material in this article are included in the article's Creative Commons license, unless indicated otherwise in a credit line to the material. If material is not included in the article's Creative Commons license and your intended use is not permitted by statutory regulation or exceeds the permitted use, you will need to obtain permission directly from the copyright holder. To view a copy of this license, visit http://creativecommons. org/licenses/by/4.0/.

\section{References}

1. I.S. Salti, M. Stiefel, J.L. Ruse, J.C. Laidlaw, Non-tumorous 'primary' aldosteronism. I. Type relieved by glucocorticoid (glucocorticoid-remediable aldosteronism). Can. Med. Assoc. J. 101 (1), 1-10 (1969)

2. D.J. Sutherland, J.L. Ruse, J.C. Laidlaw, Hypertension, increased aldosterone secretion and low plasma renin activity relieved by dexamethasone. Can. Med. Assoc. J. 95(22), 1109-19 (1966)

3. M. Stowasser, R.D. Gordon, T.J. Tunny, S.A. Klemm, W.L. Finn, A.L. Krek, Familial hyperaldosteronism type II: five families with a new variety of primary aldosteronism. Clin. Exp. Pharmacol. Physiol. 19(5), 319-22 (1992)

4. M. Choi, U.I. Scholl, P. Yue, et al., K+ channel mutations in adrenal aldosterone-producing adenomas and hereditary hypertension. Science 331(6018), 768-72 (2011).

5. U.I. Scholl, C. Nelson-Williams, P. Yue et al. Hypertension with or without adrenal hyperplasia due to different inherited mutations in the potassium channel KCNJ5. Proc. Natl Acad. Sci. USA 109 (7), 2533-8 (2012)

6. U.I. Scholl, G. Stölting, C. Nelson-Williams et al. Recurrent gain of function mutation in calcium channel CACNA1H causes earlyonset hypertension with primary aldosteronism. Elife 4, e06315 (2015)

7. L. Lenzini, S. Prisco, B. Caroccia, G.P. Rossi, Saga of familial hyperaldosteronism yet a new channel. Hypertension 71(6), 1010-1014 (2018)

8. G.T. McMahon, R.G. Dluhy, Glucocorticoid-remediable aldosteronism. Cardiol. Rev. 48(5), 682-6 (2004)

9. W.R. Litchfield, B.F. Anderson, R.J. Weiss, R.P.D.R. Lifton, Intracranial aneurysm and hemorrhagic stroke in glucocorticoidremediable aldosteronism. Hypertension 31(1 Pt 2), 445-50 (1998)

10. R.P. Lifton, R.G. Dluhy, M. Powers et al. A chimaeric 11 $\beta$-hydroxylase/aldosterone synthase gene causes glucocorticoidremediable aldosteronism and human hypertension. Nature $\mathbf{3 5 5}$ (6357), 262-5 (1992)

11. A.A. MacConnachie, K.F. Kelly, A. McNamara et al. Rapid diagnosis and identification of cross-over sites in patients with 
glucocorticoid remediable aldosteronism. J. Clin. Endocrinol. Metab. 83(12), 4328-31 (1998)

12. L. Lenzini, G.P. Rossi, The molecular basis of primary aldosteronism: from chimeric gene to channelopathy. Curr. Opin. Pharmacol. 21, 35-42 (2015)

13. J.W. Funder, Genetics of primary aldosteronism. Cardiovasc. Issues Endocrinol. 43, 70-8 (2014).

14. G.P. Rossi, G. Bernini, C. Caliumi et al. A prospective study of the prevalence of primary aldosteronism in 1,125 Hypertensive Patients. J. Am. Coll. Cardiol. 48(11), 2293-300 (2006)

15. S. Monticone, J. Burrello, D. Tizzani et al. Prevalence and clinical manifestations of primary aldosteronism encountered in primary care practice. J. Am. Coll. Cardiol. 69(14), 1811-1820 (2017)

16. C. Campino, P. Trejo, C.A. Carvajal et al. Pregnancy normalized familial hyperaldosteronism type I: A novel role for progesterone. J. Hum. Hypertens. 29(2), 138-9 (2015)

17. E. Hamilton, C. O'Callaghan, R.M. O'Brien et al. Familial hyperaldosteronism type 1 in pregnancy. Intern. Med. J. 39(2), 135-6 (2009)

18. J.A. Wyckoff, E.W. Seely, S. Hurwitz, B.F. Anderson, R.P. Lifton, R.G. Dluhy, Glucocorticoid-remediable aldosteronism and pregnancy. Hypertension 35(2), 668-72 (2000)

19. P. Mulatero, S.M.Di Cella, T.A. Williams et al. Glucocorticoid remediable aldosteronism: Low morbidity and mortality in a fourgeneration Italian pedigree. J. Clin. Endocrinol. Metab. 87(7), 3187-91 (2002)

20. V. Sanga, L. Lenzini, T.M. Seccia, G.P. Rossi, Familial hyperaldosteronism type 1 and pregnancy: successful treatment with low dose dexamethasone. Blood Press 30(2), 133-137 (2021)
21. G.P. Rossi, V. Bisogni, A useful tool to improve the case detection rate of primary aldosteronism: The aldosterone -renin ratio (ARR)-App. J. Hypertens. 34(5), 1019-21 (2016)

22. M.A. Brown, S.T.P. Thou, J.A. Whitworth, Stimulation of aldosterone by ACTH in normal and hypertensive pregnancy. Am. J. Hypertens. 8(3), 260-7 (1995)

23. G. Escher, Hyperaldosteronism in pregnancy. Ther. Adv. Cardiovasc. Dis. 3(2), 123-32 (2009)

24. R.S. Goland, S.L. Wardlaw, R.I. Stark, L.S. Brown, A.G. Frantz, High levels of corticotropin-releasing hormone immunoactivity in maternal and fetal plasma during pregnancy. J. Clin. Endocrinol. Metab. 63(5), 1199-203 (1986)

25. M. Quinkler, S. Diederich, Difference of in vivo and in vitro antimineralocorticoid potency of progesterone. Endocr. Res. 28 (4), 465-70 (2002)

26. A. Vecchiola, C.F. Lagos, C.A. Fuentes et al. Different effects of progesterone and estradiol on chimeric and wild type aldosterone synthase in vitro. Reprod. Biol. Endocrinol. 11, 76 (2013)

27. N.C. Nicolaides, A.N. Pavlaki, A.M. Maria Alexandra, et al. 'Glucocorticoid Therapy and Adrenal Suppression - Endotext NCBI Bookshelf' (2018)..

28. G.P. Rossi, V. Bisogni, G. Rossitto et al. Practice recommendations for diagnosis and treatment of the most common forms of secondary hypertension. High Blood Press. Cardiovasc. Prev. 27 (6), 547-560 (2020)

29. G.P. Rossi, V. Bisogni, A.V. Bacca et al. The 2020 Italian Society of Arterial Hypertension (SIIA) practical guidelines for the management of primary aldosteronism. Int. J. Cardiol. Hypertens. 5, 100029 (2020) 